\title{
Temperature Control of a Thermal Plasma Torch with Inductive Coupling Using the Arduino Board
}

\author{
Gustavo Fernandes de Lima, Glauco George Cipriano Maniçoba and Andrés Ortiz Salazar \\ Department of Computer Engineering and Automation, Federal University of Rio Grande do Norte, Natal 59072-970, Brasil
}

Received: February 17, 2014 / Accepted: March 11, 2014 / Published: June 25, 2014.

\begin{abstract}
Plasma torch is a device that transforms electrical energy into heat carried by a gas and its safe operation is necessary to control her temperature. This paper presents the use of the Arduino board in temperature control of a plasma torch through fuzzy control. The plasma torch of this project was built so that a flow of water can circulate through your body, allowing its cooling. The cooling system mounted consists of one radiator, one expansion vase, one water pump and one temperature sensor. The heated water coming the plasma torch is passed by the temperature sensor. This is converted in a voltage and read by an analog input port of the Arduino. This processes the information received and makes the decision to turn on/off the radiator fan and/or powered the frequency inverter water pump to control the temperature. The graph of the fuzzy control showed an oscillation between $104{ }^{\circ} \mathrm{F}$ to $122{ }^{\circ} \mathrm{F}$ around the chosen reference $113^{\circ} \mathrm{F}$. The results show that it is possible to control the temperature of a plasma torch using the Arduino board and fuzzy logic.
\end{abstract}

Key words: Arduino, torch, fuzzy logic, temperature, thermal plasma.

\section{Introduction}

Arduino is a prototyping platform electronics open source based on flexible hardware and software and easy to use. It is destined for artists, designers, hobbyists and anyone interested to create interactive objects or environments [1].

The Arduino board can integrate with environment by receiving signals inputs from types varied of sensors. And it can also affect the environment by controlling lights, motors or others actuators.

In this work, the environment of Arduino interaction was a water refrigeration system for a thermal plasma torch. The Arduino board received an electrical signal from temperature sensor and acted by controlling a frequency inverter which powered a water pump, and acted in a fan coupled a radiator.

The form how refrigeration system was projected enabled the implementation of a controller using fuzzy

Corresponding author: Gustavo Fernandes de Lima, electrical engineer, specialist, research fields: analog electronics, control systems and automation. E-mail: gustavoflima@msn.com. logic embedded on Arduino board, which controlled on spin speed of water pump and on/off fan of radiator.

The paper is organized as follows: Section 2 presents the theoretical foundation; Section 3 presents the a short description of components using in this work; Section 4 shows the results and discussion; and Section 5 presents the conclusions.

\section{Theoretical Foundation}

\subsection{Fuzzy Logic}

In 1965, the professor Lofit A. Zadeh from Berkeley's University proposed a new theory of sets, where transition of pertinence to non pertinence were slow and progressive, and no abrupt how in usual theory of sets [2]. Thus, it emerged the Fuzzy Set theory.

A system based in fuzzy logic can have your action schematized by the elements [3]: fuzzificator, rule base (knowledge base), inference engine (decision-making unit) and defuzzificator. This structure can be viewed in Fig. 1. 


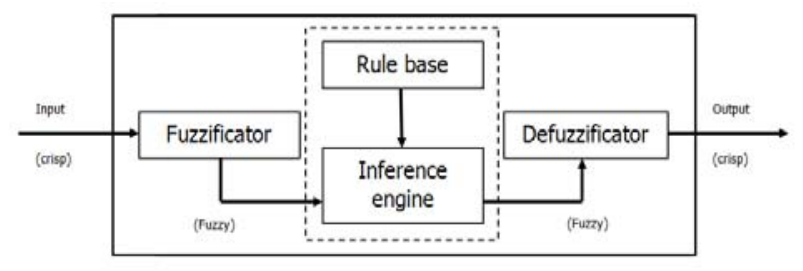

Fig. 1 Blocks of the controller fuzzy.

The fuzzificator has for function to convert the numeric values of the inputs in linguistic variables.

The rule base represents a system model to be controlled, consisting of a data base and a linguistic fuzzy rule base.

The inference engine has for feature to transform input linguistics values, with the rules, on output linguistics values.

The defuzzificator has for function to translate the output linguistic values on output numeric values, usable in a control action at real world.

\subsection{The Arduino Board}

The Arduino is a board of printed circuit $(2.68 \times$ $2.17 \times 0.39)$, indicated for creation electronics prototypes based on the philosophy of open hardware and software. It has a 01 microcontroller ATmega328P (manufactured for Atmel Company), 14 digital inputs/outputs, 06 analog inputs and pins of 3.3 Volts, 5.0 Volts and Ground (Fig. 2).

In this work, the Arduino Uno model was chosen for those reasons: it has USB connection, one jack connector for supply external, simple programming, low price and easy manipulation.

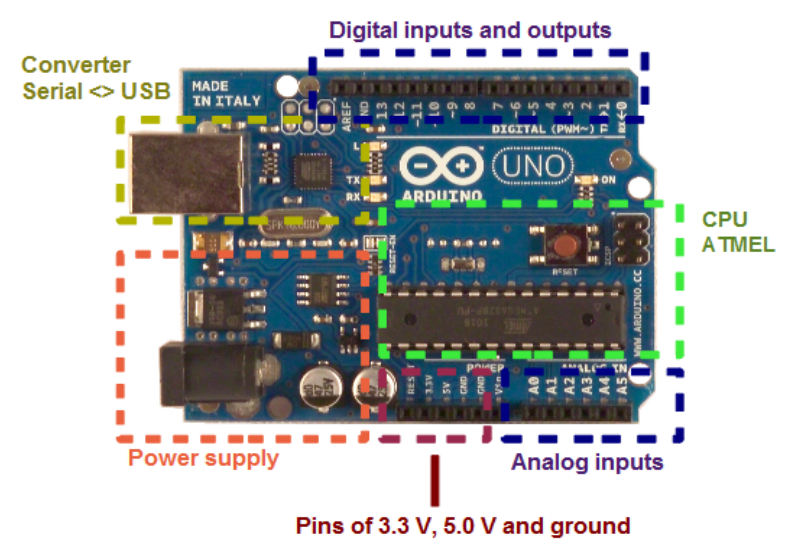

Fig. 2 Identified blocks of the Arduino Uno.
Also, two modules were used in connection with the Arduino board. The first module was SD card (Fig. 3a) and the second was LCD (liquid-crystal display) $16 \times 2$ (Fig. 3b).

In this project, the SD card module was used for save the measurements of temperature and time in txt. file. Whereas the LCD module was used to show the values of temperature and time measured.

\subsection{Temperature Sensor NTC (Negative Temperature Coefficient)}

In this project, one thermistor NTC, low cost semiconductor which decreases the electrical resistance with increment the temperature were used (Fig. 4).

The NTC sensor works for a resistance of $520 \Omega$ in $298.15 \mathrm{~K}\left(77^{\circ} \mathrm{F}\right)$ and $75.41 \Omega$ in $373.15 \mathrm{~K}\left(212^{\circ} \mathrm{F}\right)$, with a tolerance of $1 \%$ to $5 \%$, giving a non-linear graphic. For making one, this curve graphic was used in Eq. (1).

$$
R=R_{0} \cdot \exp \left[\beta \cdot\left(\frac{1}{T}-\frac{1}{T_{0}}\right)\right]
$$

where, $R$ is the thermistor resistance at temperature $T$, $R_{0}$ is a thermistor resistance at temperature $T_{0}$ and $\beta$ is the material constant which is equal to $2,864.30$ [4].

Fig. 5 presents the characteristic curve of the sensor used in this project.

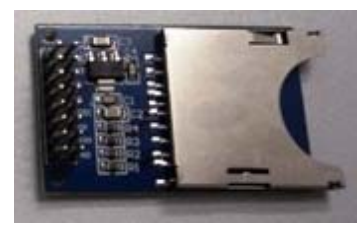

(a) SD Card

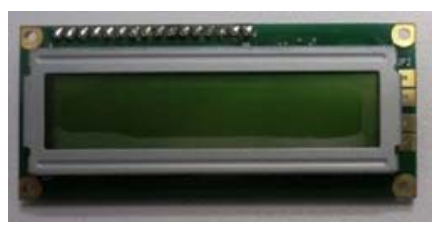

(b) LCD
Fig. 3 Modules for connection with Arduino board.

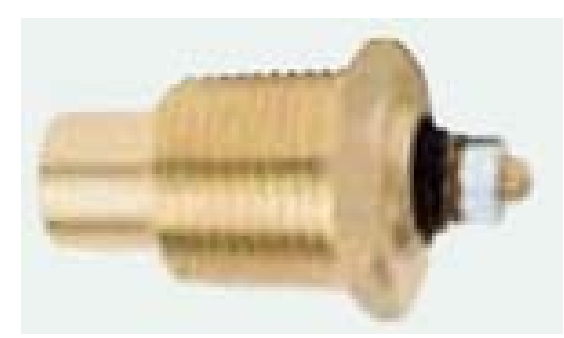

Fig. 4 NTC sensor used in this project. 


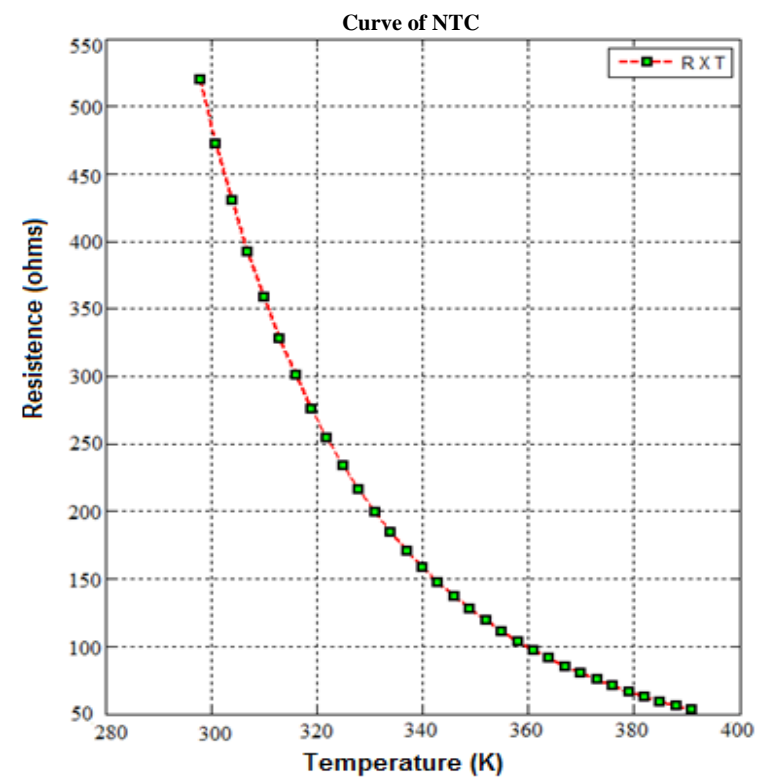

Fig. 5 Characteristic curve of the sensor NTC.

The Arduino analog input was developed to receive voltage up to $5 V_{D C}$. Therefore, it raised the need to conversion the NTC's resistance in matching voltage signal.

A voltage divider circuit was chosen to convert the resistance of NTC in measurable voltage by Arduino. In the configuration chosen, an increase of temperature was reflected in increase of voltage $V_{0}$ (Eq. (2)).

$$
V_{0}=V_{c c} \cdot\left(\frac{R_{1}}{R_{1}+R_{N T C}}\right)
$$

The $V_{0}$ voltage was connected in analog input $\mathrm{A} 0$ of Arduino board and this one has to calculate the resistance $R_{N T C}$ (Eq. (3)).

$$
R_{N T C}=\left(R_{1} \cdot \frac{V_{c c}}{V_{0}}\right)-R_{1}
$$

For determine of the current temperature of system in Kelvin, was used the Steinhart-Hart's equation (Eq. (4)).

$$
T(K)=\frac{1}{a+b \cdot \ln \left(R_{N T C}\right)+c \cdot\left(\ln \left(R_{N T C}\right)\right)^{3}}
$$

The Steinhart-Hart's coefficients $(a, b, c)$ were extracted from Ref. [5], whose values were:

$$
\left[\begin{array}{l}
a \\
b \\
c
\end{array}\right]=\left[\begin{array}{c}
0.0012 \\
0.00033151 \\
0.0000001962
\end{array}\right]
$$

In this moment, it was possible to implement the stretch of source code for Arduino able to convert the read voltage in A0 input, calculate the resistance $R_{N T C}$, determinate the temperature in Kelvin and, lastly, convert for a temperature in ${ }^{\circ} \mathrm{F}$, according to Code 1.

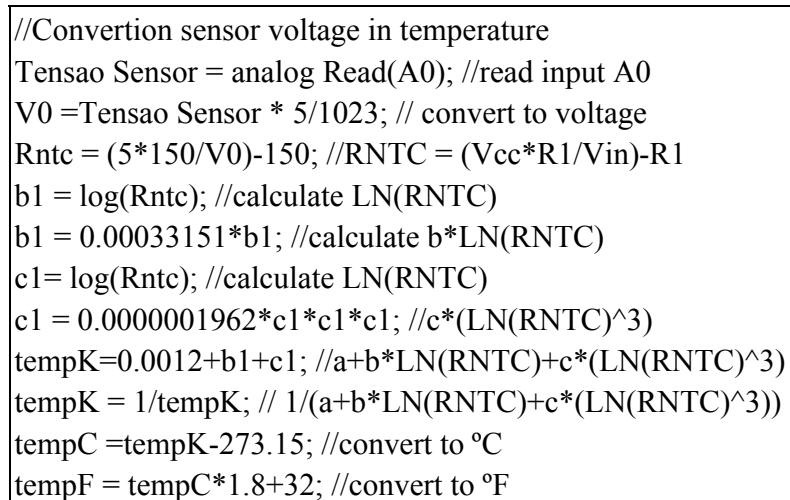

\section{Code 1 Stretch of Code for Arduino.}

\subsection{Plasma Torch}

The plasma can be defined how a gas partly ionized where exist free electrons and positive ions in movement, in the process where happen transference of energy by radiation, conduction and Joule heating between others [6].

Now, the plasma torch for Ref. [7] is a dispositive which transforms electrical energy in heat transported by a gas. With these devices, virtually either one gas can lead the plasma state.

For this project, one ICP (inductive coupled plasma) torch was chosen. According to Ref. [5], an ICP torch has by advantages do not have internal electrodes metallic, to be eroded by plasma jet, avoiding possible contamination. Fig. 6 presents one image of the ICP torch used in this project.

This torch was built to allow the water flow in two different paths, for your own cooling. Still in Fig. 6, it is possible to see an indicative numeration for these paths. In the first path, the water gets in the exhaustion opening $\left(n^{\circ} 1\right)$, goes to tube of confinement and gets 


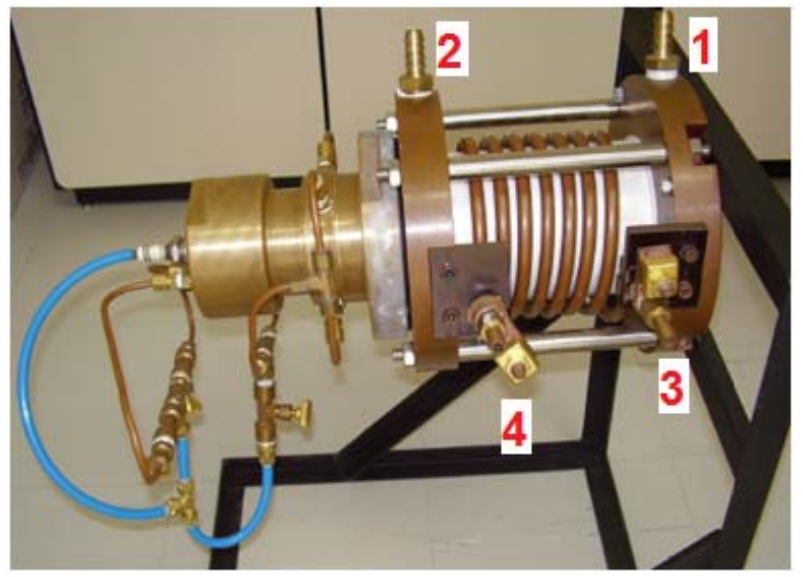

Fig. 6 ICP torch of the project.

out by the base of torch $\left(n^{\circ} 2\right)$. The second path enters in the $n^{\circ} 3$ and gets out in the $n^{\circ} 4$ by within of coil.

\section{Description of Components}

\subsection{Water Refrigeration System}

One water refrigeration system was mounted compound by one radiator (heat exchanger), which have one fan coupled and powered by three-phase motor, one expansion vase, one water pump powered by one frequency inverter and one NTC temperature sensor, according to Fig. 7.

These components have the following features in the cooling system [8]:

- Radiator: device used to exchange heat between the heated water from the plasma torch and the atmospheric air;

- Expansion vase: semitransparent plastic reservoir which allows to see the water level;

- Water pump: responsible for circulating water in the cooling system of the plasma torch;

- Temperature sensor: responsible for the measurements of water temperature in the cooling system of the torch.

\subsection{Description of Control}

The control of temperature proposal can be visualized in block diagram of Fig. 8.

In this diagram, the water temperature of system was read by Arduino. For determining the error $(\varepsilon)$ value,

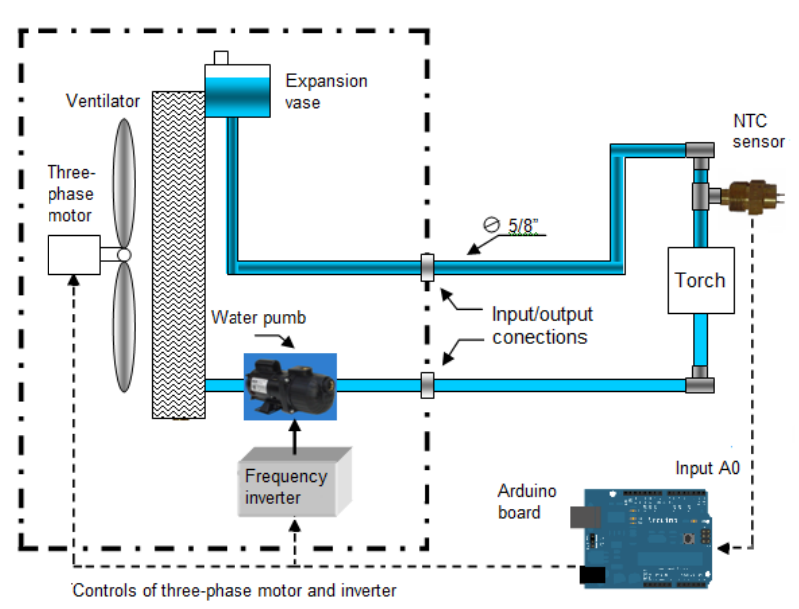

Fig. 7 Refrigeration system of the torch.

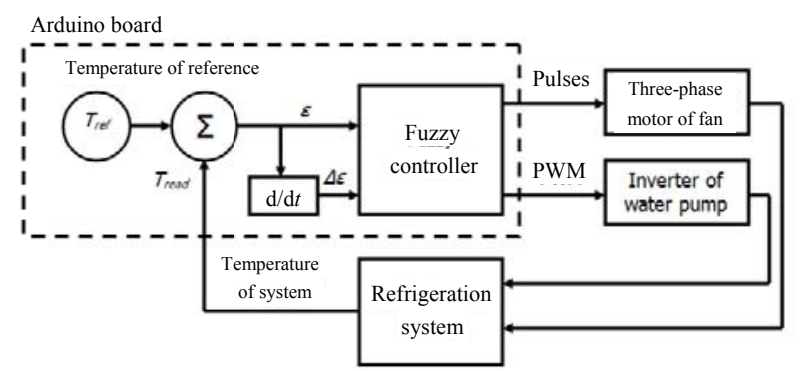

Fig. 8 Block diagram of the system.

Eq. (6) was used. This is the first variable of fuzzy controller and measurable in Celsius degree.

$$
\varepsilon\left({ }^{\circ} \mathrm{F}\right)=T_{\text {read }}-T_{\text {ref }}
$$

After that, the error value (current) was subtracted of the previous error value for determining the "variation of error" $(\Delta \varepsilon)$ value, according to Eq. (7). This is the second variable of fuzzy controller and measurable in ${ }^{\circ} \mathrm{C} / \mathrm{min}$.

$$
\Delta \varepsilon\left({ }^{\circ} \mathrm{F} / \mathrm{min}\right)=\frac{\varepsilon_{\text {current }}-\varepsilon_{\text {previous }}}{\mathrm{d} t}
$$

With two previous values, the fuzzy controller combined variables $\varepsilon$ and $\Delta \varepsilon$ in the inference rules, of type if then, and created two appropriates outputs. The first for powered the of frequency inverter and second for powered of the radiator's fan.

\subsection{Fuzzy Controller}

The fuzzy logical toolbox of MATLAB ${ }^{\mathrm{TM}}$ software was used for created the inputs variables of fuzzy system and determine output variables values.

The $\varepsilon$ variable was defined with values in $-67^{\circ} \mathrm{F}$ to 
$131^{\circ} \mathrm{F}$ and Fig. 9 shows the fuzzy set created, where linguistics values were: L (low), M (medium), SP (set point), $\mathrm{H}$ (high) and VH (very high).

The "variation of error" variable was defined with values in $22.1^{\circ} \mathrm{F} / \mathrm{min}$ to $41.9^{\circ} \mathrm{F} / \mathrm{min}$ and Fig. 10 shows the fuzzy set created, where linguistics values were: FF (falling fast), FS (falling slow), S (stable), RS (rising slow) and RF (rising fast).

The first output variable was called PWM (pulse width modulation) and represented the signal of powered of the frequency inverter ( 0 to $100 \%$ ) of water pump and Fig. 11 shows the fuzzy set created, where linguistics values were: $\mathrm{S}$ (small), $\mathrm{M}$ (medium), B (big) and VB (very big).

The second output variable was called pulses and represented the on/off signal of radiator's fan and Fig. 12 shows the fuzzy set created, where linguistics values were: off and on.

For inference machine, the Mandani was used and the defuzzification was calculated using the medium of the maxima pertinence.

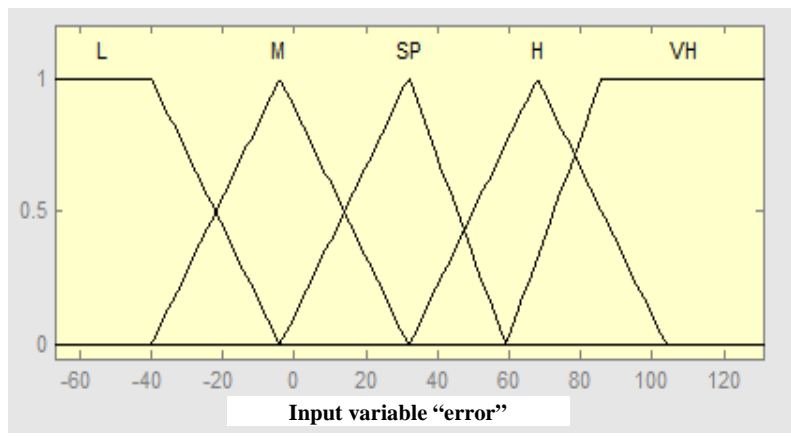

Fig. 9 Pertinences of the "error" variable.

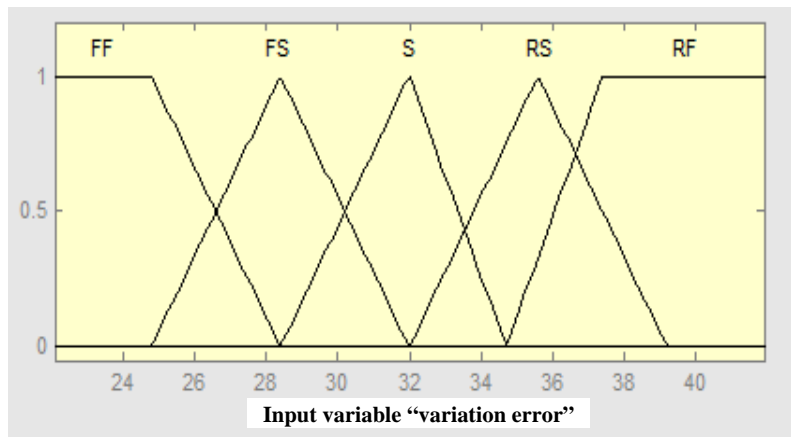

Fig. 10 Pertinences of the "variation of error" variable.

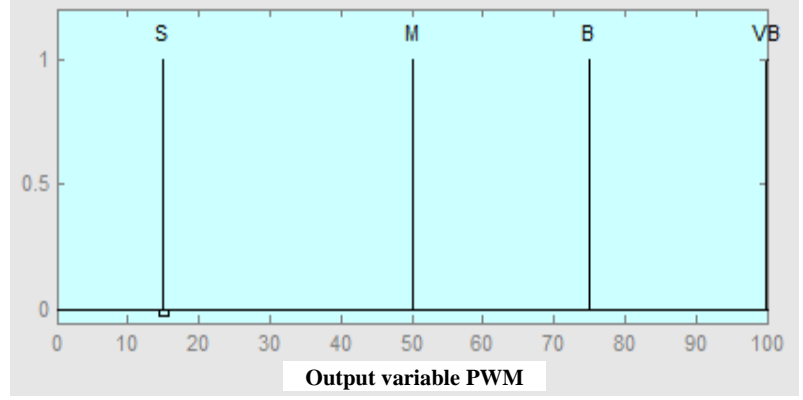

Fig. 11 Pertinences of the PWM variable.

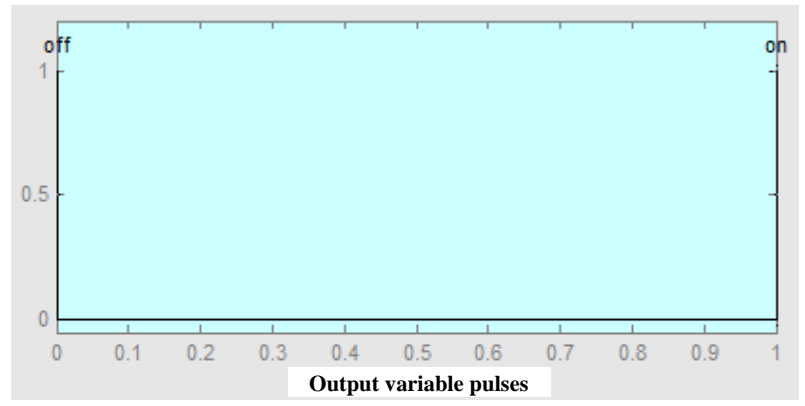

Fig. 12 Pertinences of the pulses variable.

\section{Results and Discussion}

For the demonstration of the control operation, five real experiments were made. In all, the reference temperature was configured in $113{ }^{\circ} \mathrm{F}$, for security reasons. The radiator's fan temperature powered was configured in $122^{\circ} \mathrm{F}$ and after was turned off when the temperature achieved $104^{\circ} \mathrm{F}$.

The fuzzy controller was configured for keeping the PWM variable in a constant value. In the first experiment, that value was $15 \%$ (green curve), in second $50 \%$ (yellow curve), in third $75 \%$ (red curve) and in fourth $100 \%$ (black curve). In the last experiment, the fuzzy controller was configured for any PWM variable value, in range from 0 to $100 \%$ (blue curve).

Fig. 13 shows all curves of the five real experiments. This allowed to see the temperature behavior when the PWM variable was remained constant and when her changed freely. In all, the fuzzy controller worked how expected and allowed the temperature variation was within of the range previous determined from $104^{\circ} \mathrm{F}$ to $122^{\circ} \mathrm{F}$. 


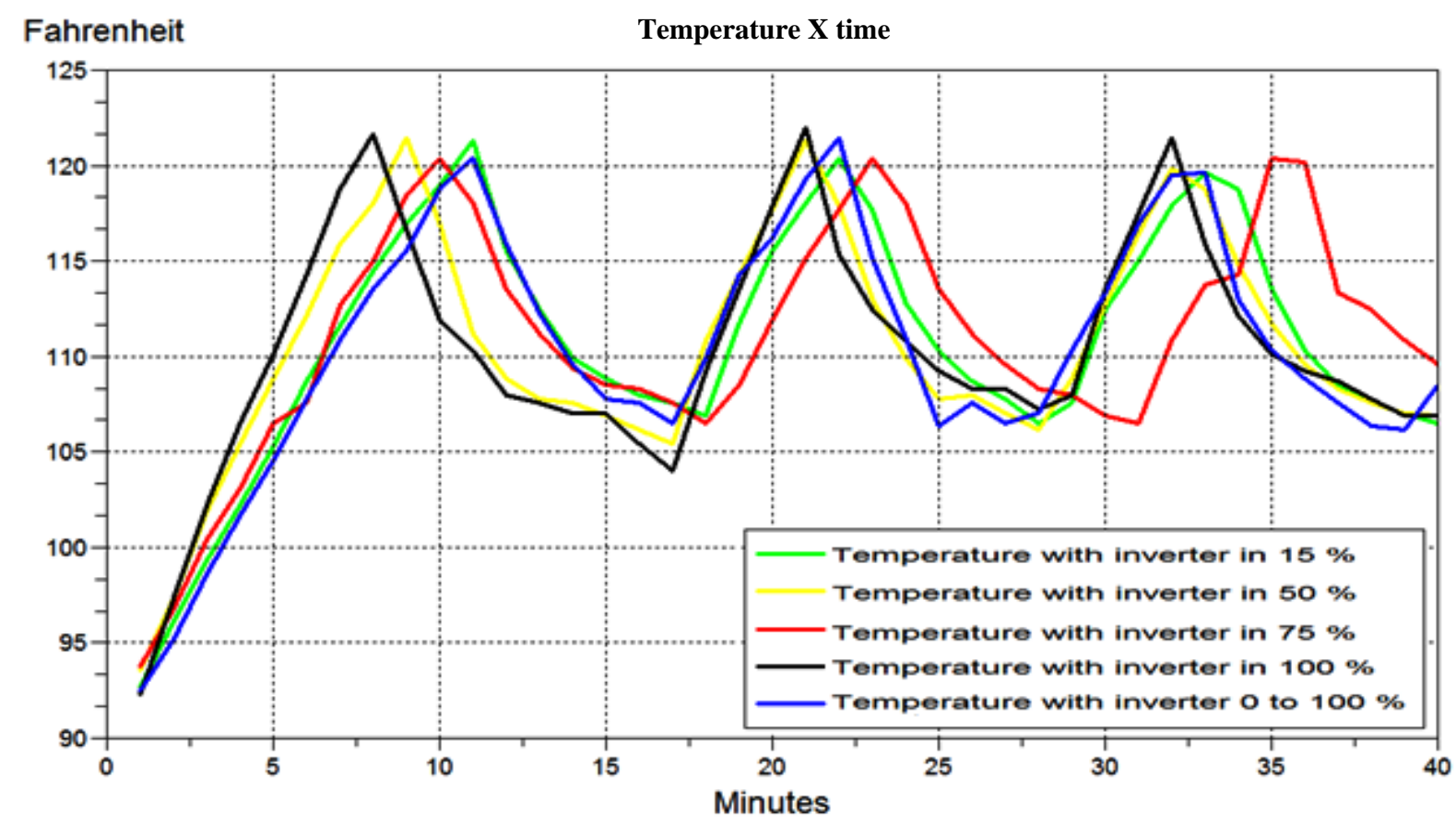

Fig. 13 Variation of the water temperature.

\section{Conclusions}

In this work, it presents the use of Arduino board in temperature control of water which cools a thermal plasma torch used the fuzzy control. The objective was achieved, once the temperature of water ranged between $106.16{ }^{\circ} \mathrm{F}$ and $121.46{ }^{\circ} \mathrm{F}$ within the range from $104{ }^{\circ} \mathrm{F}$ to $122^{\circ} \mathrm{F}$ as previous defined. This result was possible why the temperature is a magnitude slow varies, which allowed for Arduino board works without big computational endeavor.

\section{References}

[1] Arduino Home Page, http://www.arduino.cc (accessed 2013).

[2] M. Bilobrovec, R.F.M. Marçal, J.L. Kovaleski, Implementation of an intelligent control system using fuzzy logic, in: XI SIMPEP, Bauru, SP, 2004.

[3] F.M.L. Filho, H.L. Gosmann, A. Bauchspiess, Fuzzy control for liquid level system, in: XIV Congresso Brasileiro de Automática, Natal, RN, 2002, pp. 3017-3022.

[4] G.G.C. Maniçoba, Temperature control of the water cooling system of a plasma torch using fuzzy logic, Master Thesis, Federal University of Rio Grande do Norte, 2013.

[5] G.G.C. Maniçoba, A.O. Salazar, J.A.D. Amado, J.A.B. de Oliveira, J.P. Dubut, A. S. Lock, et al., Design and simulation of a thermal plasma torch with inductive coupling, in: VII CONEM, São Luiz, MA, 2012.

[6] M.F.G. Rosias, Emission Atomic Spectrometry with Inductively Coupled Plasma, 1st ed., CENA, Piracicaba, SP, 1998.

[7] N.M. Nishio, A. Takabatake, Energy Production from Waste of a Water Reuse, Graduation Project, University of São Paulo, São Paulo, SP, 2002.

[8] A.M.F. Guimarães, Development of a control system for a plant of waste blanketing by plasma, Ph.D. Thesis, Federal University of Rio Grande do Norte, 2009. 